\title{
L'évolution du suffixe -issime : un inventaire et une fréquence des formes attestées dans Frantext
}

\author{
Anders Bengtsson (Stockholm University, Sweden)
}

\begin{abstract}
The synthetic superlative -ÍSSIMUS in Latin survived in Italian, whereas it was borrowed in the Romance languages on the Iberian Peninsula and in French during the Renaissance. This suffix has been very frequent in these languages with the exception of French. In this language it has been accepted merely when used in titles. Condemned by grammarians, the suffix has thus been quite rare in French literature. The present study shows however that in the database Frantext, which comprises mostly literary texts, nearly 1,400 occurrences of words with the suffix -issime are found, rarissime and richissime being the most frequent (apart from titles). But with the emergence of new media, it seems that the suffix has become much more frequent in French. These adjectives are found mainly in areas like politics, sports, travels, adult movies and in comments by web visitors as shown in this study.
\end{abstract}

Keywords: suffix, superlative, morphology, French, Romance languages

\section{Introduction}

Parmi les suffixes qui connaissent un succès en français depuis un certain temps, surtout dans la langue parlée ou la langue écrite informelle, -issime occupe une place particulière. Noailly $(1999,33)$ parle de resurrection, ce qui est un terme bien adapté à la situation actuelle. Or, à la différence du suffixe $-o s$, étant une nouveauté pour le français, également présent dans la langue parlée et la langue écrite informelle, -issime apparaît dès la Renaissance, époque où les suffixes -esque et -issime deviennent productifs en français d'après Siouffi (dans Rey, Duval et Siouffi 2007, 532). Comme le suffixe latin -ÍSSIMUS, marquant un haut dégré, aurait abouti à -esme en ancien français, on comprend dès lors qu'il s'agit d'un emprunt. D'après Elcock (1960: 71), ce sont les savants médiévaux qui ont restauré le suffixe latin -ÍSSIMUS, ce dont témoignent les produits -isme, -ísimo et -issimo en français, espagnol et italien médiévaux. Ce qu'on relève en ancien français, ce sont justement quelques adjectifs en $-i s m e^{l}$, de formation savante, illustré par les trois exemples suivants, tirés d'œuvres bien connus :

(1) Ne s'en corucet giens cil saintismes hom (v. 268, Vie de saint Alexis)

(2) Pois sunt muntet sus el palais altisme (v. 2708, Chanson de Roland)

(3) Un grandisme nes plat (24,18, Aucassin et Nicolette)

\footnotetext{
${ }^{1}$ Cela rappelle les formes en catalan: santisme et altisme (Griera 1965: 154).
} 
Cependant, ce superlatif absolu disparaît plus tard de l'ancienne langue. Mais il n'est pas sans intérêt de s'attarder au provençal, car les occurrences citées par Hultenberg (1903, 10) sont presque identiques à celles qu'on relève en ancien français :

(4) Benezectes sias, filh de Dieu altisme, que doniest a nos repaus de .I. dia e .II. nueitz (Appel $1930: 117,81-82^{2}$ )

(5) En lui ac chavalier moltisme bo (Roman de Girart de Rossillon, fol. 14)

(6) Car per la crotz sanctisma, sia sens o foldatz, nos irem per la gata, si vos o comensatz (Appel 1930: 7, 462)

Mahn $(1885,291)$ mentionne à titre d'exemple altisme, mais aussi les exemples suivants dans sa grammaire : carisme (de CARÍSSIMUS), prosme et plusieurs variantes graphiques du produit de PRÒXIMUS, moltisme, santisme et enfin pesme. Il n'est pas dû au hasard que les occurrences en français et en provençal se ressemblent tant ; en plus, ces exemples sont les plus récurrents dans les textes, à l'exception de moltisme peut-être, qui ne semble pas attesté en ancien français. Or, c'est plus tard, sous la double influence du latin et surtout de l'italien, qu'apparaissent en français un grand nombre de superlatifs en -issime d'après Nyrop $(1902,336)$, mais qui seront par la suite condamnés par les grammairiens ${ }^{3}$. Pour Hultenberg $(1910,10)$, il s'agit d'un emprunt à l'italien.

Après ces préliminaires, nous ferons d'abord un survol de la situation de ce suffixe dans les langues romanes pour ensuite rendre compte de la situation en français. Dans la partie empirique, nous relèverons les occurrences dites littéraires dans la base de données Frantext et quelques occurrences contemporaines dans la langue écrite informelle afin d'établir une comparaison typologique.

\subsection{Le produit du suffixe -ÍSSIMUS dans les langues romanes}

Dès le latin classique, le superlatif absolu était un moyen commode pour renforcer le sens des adjectifs (Hultenberg 1903, 9), mais c'est surtout en bas latin que le suffixe ÍSSIMUS abondait. Comme il revêtait un caractère savant, il s'est imposé par l'influence des lettrés, pendant que le peuple ne s'en servait pas selon Hultenberg $(1903,9)$. L'italien s'en est toujours servi à l'en croire Hultenberg, usage qui est passé dans la péninsule hibérique, tout en préservant le caractère savant. Ce suffixe est regardé comme plus énergique que le renforcement par adverbes d'après Hultenberg (1903, 10). Ainsi, dans presque toutes les autres langues romanes, le superlatif -issime est beaucoup plus fréquent qu'en français, à des dégrés variés. Nous commencerons par l'italien, où on relève non seulement des superlatifs formés sur des adjectifs tels que dolcissimo ("très doux"), sur des adverbes tels que benissimo ("très bien"), mais aussi sur des participes passés ainsi qu'il ressort de cet exemple tiré de la grammaire de Bach \& Schmitt Jensen (1995, §526), où vendutissimo correspond à "très vendu":

(7) Il suo pamphlet è vendutissimo.

\footnotetext{
${ }^{2}$ Dans le même texte, on relève aussi Merce, filh de Dieu altisme! (117,68-69). Dans un autre texte, on relève la variante graphique autisme $(107,22)$.

${ }^{3}$ Voir également Hultenberg $(1903,9)$.
} 
Ainsi, le fait d'ajouter ce suffixe confère un sens intense et énergique au mot de départ. Les possibilités d'ajouter ce suffixe à des mots de différentes catégories grammaticales sont visiblement multiples en italien. La souplesse est en outre illustrée par des exemples où on ajoute le suffixe à des pronoms indéfinis tels que stessissimo (un superlatif formé sur l'équivalent de lui-même) et nessunissmo (formé sur l'équivalent de personne) ; la grammaire de Bach \& Schmitt Jensen signalent même campionissimo, formé sur le substantif campione ("champion"), néologisme des années 60, désignant le cycliste fort célèbre Angelo Fausto Coppi.

C'est aussi en italien qu'on en a les plus anciennes attestations d'après Serianni (2008, 27) ; c'est d'ailleurs la seule langue romane où ce suffixe est fréquent dès le Moyen Âge. Pour Bourciez (1967, 522), ces superlatifs sont des emprunts savants, mais ayant été réintroduits tôt dans l'usage populaire, ils sont restés très vivants. Si nous en venons en espagnol, le suffixe paraît presque inconnu dans l'ancienne langue (Menéndez Pidal 1968, 221), car cette forme savante semble peu usitée au Moyen Âge. On rencontre à cette époque par exemple dulcísimo ("très doux)" et altísimo ("très haut") et parfois, on ajoute le superlatif -ísimo à la forme latine de l'adjectif en espagnol (Menéndez Pidal 1968, 221) ; c'est ainsi qu'on relève antiquísimo, crudelísimo, amabilísimo, acérrimo, paupérrimo. Plus tard, le superlatif s'y est introduit au $\mathrm{XV}^{\mathrm{e}}$ siècle sous l'influence de l'italien (Bourciez 1967, 447). Aujourd'hui, le suffixe peut être ajouté à de nombreux adjectifs selon Butt et Benjamin $(2011,59)$, mais pas à tous ${ }^{4}$. Il faut également retenir que les participes verbaux purs et durs n'acceptent pas le suffixe selon Bosque et Demonte $(1999,302)$, prenant comme exemple *Lo han estudiadísimo à l'opposé de Está estudiadísimo. L'exemple suivant, où l'adjectif correspond à "très étudié", est donc tout à fait possible en espagnol :

(8) Un asunto estudiadísimo por los especialistas ${ }^{5}$

Parmi les formes irregulières, souvent de formation savante, on peut également noter cursilísimo (formé sur cursi, "affecté"), jovencísimo (joven, "jeune") et lejísimo (lejos, "lointain") (Butt et Benjamin 2011, 60). Dans une large mesure en espagnol, il s'agit donc d'adjectifs de formation savante, ce qui vaut également pour le portugais.

Or, à première vue, la situation diffère dans cette langue, car même trois variantes du suffixe existent : -érrimo, -ílimo et -íssimo. Raposo et Vicente (2013, 1430), donnent comme exemple aquele poeta é celebérrimo, o meu curso é dificílimo et este filme é interessantíssimo. Selon Kröll $(1963,245)$, le premier est très rare, même à l'écrit, peut-être dû à son caractère latinisant, comme dans magérrimo et paupérrimo ; or, ces derniers adjectifs appartiennent aux formes qui sont souvent remplacées par des formes populaires telles que pobríssimo. Le second, -ílimo, n'existe pratiquement que dans deux formes, à savoir facílimo et difícilimo (Kröll 1963, 245). Ainsi, il paraît que seul le suffixe -íssimo prévaut s'il n'est pas remplaçé par la forme analytique avec muito. Said Ali signale à ce sujet que, pour exprimer le dégré intense d'un adjectif, le portugais peut avoir recours non seulement à -íssimo, mais aussi à muito, extraordinariamente, extremamente etc. (1966, 82). Ce superlatif est, tout comme dans l'espagnol, de formation savante (Said Ali 1966, 82 ; cf. également Williams $1938, \S 128.2)$ et c'est pendant la Renaissance que le suffixe commence à être fréquent en portugais (Kröll 1963, 247), car avant, on utilisait plutôt la forme analytique avec mui (muito).

\footnotetext{
${ }^{4}$ Voir la liste sous 4.9.2 chez Butt et Benjamin (2011).

${ }^{5}$ Exemple (203b) chez Bosque et Demonte (1999, 302).
} 
Un superlatif qui paraît très fréquent à la Renaissance est grandíssimo, qu'on relève, selon Said Ali $(1966,83)$, chez les auteurs du XVI ${ }^{\mathrm{e}}$ et XVII ${ }^{\mathrm{e}}$ siècle. Autre remarque qui intéresse la nature de ce suffixe concerne les affinités du portugais avec l'italien en ce qui concerne sa souplesse, étant donné qu'on peut l'ajouter à des pronoms indéfinis tels mesmo et tanto, ce que nous venons de voir à propos de l'italien. Kröll cite à ce propos mesmíssimo et tantíssimo (1963, 248-249) et même la forme campíonissimo, formée sur un substantif (Kröll 1963, 251), ce qui rappelle la situation en italien. Il convient également de remarquer qu'on relève les formes semi-savantes dans des dialectes portugais telles que -íssemo, -essíssemo et -ismo d'après Williams $(1938, \S 128.2)$. Parfois, on relève même des superlatifs basés sur des formes populaires affublées du suffixe savant : ainsi, on se sert de docíssimo au lieu de dulcíssimo.

Si nous en venons au catalan, deux variantes du suffixe semblent exister, ce qui rappelle la situation en portugais : -íssim et -èrrim d'après Cabré (dans Solà, Lloret, Mascaró et Pérez Saldanya 2002, 759) qui donnent comme exemples amplíssim, facilíssim, celebèrrim et paupèrrim. On utilise ce suffixe de la même manière que dans les autres langues ibéroromanes, à une exception près, car un adjectif affublé du suffixe peut constituer la base d'un adverbe (Wheeler, Yates \& Dols 1999, 104), ce qui ne semble pas être attesté ailleurs. Ici, dans notre exemple, emprunté à cette grammaire, le superlatif rectíssimament équivaut à "d'une façon très correcte":

\section{(9) Va educar els seus infants rectíssimament.}

Par ailleurs, dans les dialectes des Baléares, on remarque une grande souplesse en ce qui concerne l'usage du suffixe ; en fait, il peut être ajouté à des adverbes tels que bé $\rightarrow$ beníssim ("très bien"), aviat $\rightarrow$ aviadíssim ("très rapidement"), voire à des expressions adverbiales telles que a prop $\rightarrow$ a propíssim ("très proche") (Wheeler, Yates \& Dols 1999, 104), ce qui paraît un trait propre à ces dialectes autant que nous sachions. Pour finir, tout comme en espagnol et en portugais, on a raison de croire que le suffixe est également d'origine savante en catalan ; parmi les exemples cités par Badía Margarit $(1951,260)$ on remarque dolcíssim, santíssim, sacratíssim et gravíssim.

Il ressort de ces préliminaires que quatre langues romanes, à savoir le français, l'espagnol, le portugais et le catalan se ressemblent, formant un groupe à part, où le suffixe, d'origine savante, a été emprunté pendant la Renaissance. Mais, comme on va bientôt le voir, le français y fait exception à plus d'un titre. Il nous reste cependant une autre langue romane, le roumain, où le produit du suffixe -ÍSSIMUS est en principe absent. Si l'italien est la langue qui tolère à un très haut dégré les superlatifs absolus en -issime depuis longtemps, la chose diffère de beaucoup en roumain pour des raisons évidentes ; l'explication réside dans le fait que la naissance du roumain est postérieure à la Renaissance, étant donné que la langue littéraire roumaine est née au XVII ${ }^{\mathrm{e}}$ siècle (Elcock 1960, 485). Certes, Serianni fait à ce sujet mention des occurrences très rares en roumain : rarisim et urgentisim $(2008,28)$, mais, où, tout comme dans tant d'autres cas, l'italien a servi de modèle. Ainsi, le roumain préfère depuis longtemps les formes analytiques foarte rar et foarte urgent respectivement ("très rare" et très urgent"). La fréquence du suffixe -issime n'est donc pas uniforme dans la Romania. 


\subsection{L'histoire du suffixe -issime en français}

Pour retourner à l'histoire du suffixe dans la langue française, celui-ci reviendra au cours de la Renaissance en tant qu'emprunt. D'après Brunot (1906, 306), il se peut que la Pléiade soit derrière de cette forme ; en effet, Ronsard avait dans son Art poétique (VI, 462), promis aux poètes de leur montrer à composer des noms superlatifs entre autres. Or, Brunot ne croit pas trop à cette origine, mais parle de Peletier du Mans qui était favorable à ces formes, citant grandissime et belissime chez lui. Brunot parle plutôt de la mode italienne (1906, 306), ce qui est la raison pour laquelle ces formes apparaissent chez Rabelais qui s'en sert ironiquement : verissime, perfectissime. On relève chez Noel du Fail, toujours selon Brunot $(1906,306)$, scientissime $(1,128)$, beatissime $(1,54)$ et chez Larivey bestialissime, des formes sans lendemain.

Mais on ne manquera pas de noter que le suffixe provoquera des réactions de la part d'Henri Estienne qui accusa la mode italienne d'en être responsable :

Et à propos de ce Grandissime dont je vien d'user, notez que ces superlatifs sont maintenant fort plaisants aux courtisans comme sonnans fort bien, et ayans quelque garbe: tellement qu'il vous faudra prendre garde de dire plustost Doctissime, que tresdocte : plustost Bellissime, que tresbeau : plustost Bonissime, que Tresbon. (Estienne, Dial., 1, 285).

Si la réaction de Meigret est aussi hostile que celle d'Estienne, se manifestant ainsi chez ce grammairien, selon qui le français ne peut pas digérer les superlatifs en -issime :

Ao regard de la nouuell' inuencion des Superlatifs latins en ssime: com' illustrissime, Reuerendissime, Inuictissime, qe nou' pouuons appeller superlatifs tituleres, l'uzaje de la lange Françoeze ne le' peut goutter: e encores moins dijerer. (Meigret 1550, 38)

il ne manque pas de nous fournir un renseignement morphologique utile :

Ao demourant je confesse bien qe noz ançetres ont tyré des superlatifs Latins: come, maxime, minime: més il ne gardet pas la sinificaçion superlative. E pourtant pour dire q'un hom' e tre' grant, nou' ne dizons pas, q'il et maime, ne en semblable de minime. (Meigret 1550, 38-39)

Il semble donc mieux accepter les superlatifs, où le sens intense a disparu. Parmi les grammairiens, Jean Pillot (1561) paraît plus indulgent ; dans ces cas, l'usage de la cour est considéré comme étant correct :

Quelques-uns, dit-il, voulant enrichir notre langue, lui donnent un superlatif à l'imitation des Latins; ils disent pour tres sçavant, sçavantissime ; pour tres bon, bonissime ; pour tres reverend, reverendissime. Ces formes sont dues à la cour, dont l'autorité est telle qu'il vaut mieux se tromper avec elle que de bien parler avec les autres, et que l'on a toujours raison avec ce mot : "elle l'a dit". (Livet 1859, 292-293)

Le siècle suivant tolérera les adjectifs en -issime, en tout cas dans certains domaines, étant donné que les grammairiens se résignent à laisser les dignitaires d'Eglise s'en servir. Le grammairien Bernhard accepte ainsi à titre d'exemple serenissime, illustrissime, reuerendissime, grandissime, alors que Maupas ne tolère que grandissime et, dans une certaine mesure, doctissime, tout en emettant cette réserve à propos de la titulature : 
Grandissime, est assez receu pour Tresgrand. Et quelquefois Doctissime. Autres superlatifs de cettte sorte escorchez du Latin, ne sont gueres recevables: Sauf qu'és tiltres \& inscriptions adressees aux grands, on usurpe Illustrissime, Serenissime, Reuerendissime. Nommément au Prelats d'Eglise. (Maupas 1618, 50)

Employé dans les titres honorifiques, le suffixe paraissait donc plus accepté que dans d'autres cas et si nous continuons d'examiner ces dernières formes, qui semblent très fréquentes (voir le chapitre suivant), Dubois et Dubois-Charlier $(1999,165)$ précisent que le suffixe est issu du latin et de l'italien, où il relève de la titulature. C'est peut-être la raison pour laquelle ces occurrences ont été et sont toujours mieux acceptées que d'autres. Le suffixe fait par conséquent fonction d'amélioratif d'adjectifs ; Dubois et Dubois-Charlier notent également que les termes de la titulature ont été francisés, à titre d'exemple généralissime, ce que nous verrons par la suite, et parfois, ces termes sont employés ironiquement comme dans l'exemple : l'illustrissime général Tartarin (Dubois et Dubois-Charlier 1999, 165). On notera que l'antéposition de ces termes semblent de règle, ce qui ne surprend peut-être pas.

À part ces termes d'étiquette, certains superlatifs appartiennent à la langue familière comme rarissime et richissime, d'autres à la langue plaisante comme ignorantissime et savantissime (Nyrop 1902, 337) et parmi les occurrences célèbres au cours de l'histoire de la langue française de caractère ludique, on peut citer une occurrence provenant de la comédieballet Le mariage forcé de Molière, où ignorantissime fait partie d'une cascade d'adjectifs formés sur ignorant :

(10) "Oui, je te soutiendrai par vives raisons que tu es un ignorant, ignorantissime, ignorantifiant, et ignorantifié par tous les cas, et modes imaginables." (Molière, Le mariage forcé, 1668, 31).

Quant à l'usage de ces superlatifs au XVII ${ }^{\mathrm{e}}$ siècle à la Cour, on peut relever un commentaire intéressant fait par Bouhours avec trois exemples, dont habilissime paraît fort rare. La remarque de Siouffi selon qui la conversation ne fait pas tout le bon usage $(2013,82)$ est bien pertinente à cet égard, ce qui ressort du passage suivant :

Ces superlatifs se disent dans le discours familier, \& les gens de la Cour en usent souvent. Quand on leur demande si un homme est habile, ils répondent habilissime. On dit, il a fait une grandissime fortune; elle est belle, bellissime; ce livre est rare, rarissime. Tout cela ne s'écrit point, \& ne se dit point en public ; \& il n'y a gueres d'apparence que ces superlatifs, qui sont contre le génie de nostre Langue, entrent jamais dans les livres; c'est bien assez pour eux d'estre soufferts dans la conversation. (Bouhours 1675, 218)

La suite de ce commentaire intéresse à un haut dégré le sujet de cette étude, étant donné que Bouhours ajoute que les autres langues romanes, à savoir l'italien et l'espagnol, sont pleines d'adjectifs de ce genre à l'opposé du français :

Les Italiens \& les Espagnols ont en cela de grands avantages sur nous; si c'en est un d'estre riche en superlatifs, \& et d'avoir la liberté de s'en servir quand on veut. Leurs Langues sont pleines de ces termes propres à exagérer les choses, \& leurs livres en sont remplis. (Bouhours 1675, 218).

Comme le constate Siouffi (2010, 376), tous les commentateurs postérieurs à Vaugelas admirent de façon ambigue l'innovation linguistique : ambigue parce qu'ils veulent la réduire 
et l'expliquer. À l'égard du suffixe -issime, on peut dire que les locuteurs dépassent largement le maigre savoir des grammairiens.

Qu'en est-il aujourd'hui dans les grammaires contemporaines ? Une grammaire comme celle de Grevisse note la même chose que nous avons déjà pu constater : le suffixe marquera un haut dégré dans des termes d'étiquette tels que excellentissime et illustrissime, ainsi que dans rarissime et richissime et « dans des formations occasionnelles, parfois plaisantes, de la langue littéraire » $(2001, \S 555)$.

Plus négative, la réaction de l'Académie française, normative, montre encore une fois que le suffixe-issime n'est pas tout à fait accepté dans la langue française :

La formation de termes à valeur superlative à l'aide du suffixe-issime, emprunté à l'italien et, à travers lui, au latin, est à la mode. Si Bellissime, Richissime, Élégantissime sonnent comme de plaisants italianismes, le procédé marque une inutile emphase lorsqu'on applique ce suffixe à des termes dont le sens, très fort, n'appelle pas de superlatif. Ainsi Génialissime, Sublissime (ou sublimissime), Urgentissime sonnent tout simplement « ridiculissime ${ }^{6}{ }^{6}$

Nous pouvons donc constater, en guise de conclusion sur le cas du français, que les jugements de la part des grammairiens ont été le plus souvent négatifs, l'exception étant les titres honorifiques. On verra par la suite si ces occurrences illustrent bien la situation dans la langue moderne.

\section{Inventaire Frantext}

Étant donné que les jugements dans les grammaires sur le suffixe-issime ont été plutôt négatifs au cours des siècles, on peut se demander dans quelle mesure et dans quels textes il est attesté. Nous travaillerons selon l'hypothèse que la plupart des occurrences appartiendront aux titres honorifiques, puisqu'ils paraissent mieux acceptés que les autres. Dans le deuxième volet de cette étude, nous ferons par conséquent un inventaire des occurrences relevées dans le corpus Frantext. Cette base de données importante, toujours agrandie et renouvelée, nous permet de voir le contexte dans lequel se trouve l'occurrence en question. Ainsi, il est souvent possible de comprendre la raison exacte de l'usage du mot, car une étude qualitative est aussi intéressante qu'une étude quantitative. Tout d'abord, nous donnerons une liste répertoriant les adjectifs les plus fréquents dans la littérature française relevés dans Frantext. ${ }^{7}$ Au total, nous avons pu relever 1384 occurrences dans cette base de données lors de notre consultation. Peu surprenant, si nous examinons le palmarès pour ainsi dire, les titres honorifiques (généralissime, sérénissime, eminentissime, révérendissime, excellentissime avec leurs variantes graphiques respectives) occupent presque la moitié des occurrences (533), soit $38 \%$ :

1. 340 occurrences: 308 généralissime, 16 géneralissime, 16 generalissime

2. 181 occurrences: 147 sérénissime, 33 serenissime, 1 sérenissime

3. 152 occurrences: rarissime

\footnotetext{
${ }^{6}$ Sous la rubrique Dire, ne pas dire (le 2 février 2012) : http://www.academie-francaise.fr/issime-superlatif-en

${ }^{7}$ Consulté en janvier 2018.
} 
4. 106 occurrences: 84 eminentissime, 22 éminentissime

4. 106 occurrences: 73 révérendissime, 33 reverendissime

6. 102 occurrences: richissime, rigissime ${ }^{8}$

7. 87 occurrences: grandissime

8. 57 occurrences: illustrissime

10. 37 occurrences: amarissime

11. 25 occurrences: excellentissime, tresexcellatissime

12. 16 occurrences: gravissime

13. 5 occurrences: bellissime, ignorantissime, longuissime, maréchalissime, purissime

18. 4 occurrences: clarissime, doctissime, nullissime, sainctissime, simplissime, specialissime

24. 3 occurrences: brillantissime, chérissime, dulcissime, énormissime (2)/enormissime, finissime, narcissime, novissime, sanctissime, savantissime, vérissime (2)/verissime

34. 2 occurrences: brévissime, capitalissime, carissime, célébrissime, christianissime, dilectissime, élégantissime, intimissime, légérissime, modernissime, profondissime, récentissime/recentissime, strenuissime, urgentissime, vaillantissime, vastissime

50. 1 occurrence: amplissime, antiquissime, ardentissime, argutissime, bièvrissime, bonissime, bourgeoissime, bravissime, brunissime, bulozissime, caniculissime, celsissime, certissime, classisssime, colendissime, constantissime, discrétissime, faussissime, favorissime, folissime, fortissime, fourbissime, habilissime, honnêtissime, idiotissime, importantissime, invictissime, josephissime, louchissime, madrilenissime, mochissime, nervosissime, dix-neuvièmissime, noblissime, pâlissime, parfaitissime, pauvrissme, perfectissime, pianissime, plénissime, présentissime, prudentissime, promptissime, purrissime, radicalissime, raidissime, rapidissime, réalissime, ridiculissime, rosissime, sapientissime, scélératissime, sobrissime, sublimissime, sublissime, suzanissime, tendrissime, terribilissime, timidissime, unitissme, universalissime, véhémentissime, vieillissime, vivantissime.

Les adjectifs qui sont employés comme des titres honorifiques que nous venons de citer n'appellent pas de remarques, car ils apparaissent presque toujours devant un terme de titulature et sont presque toujours antéposés, ce qui était attendu. Or, pour généralissime, on peut remarquer quelques exemples qui font fonction de substantif, dont nous reproduisons celui-ci :

(11) La vérité, elle est dans les prêches du moine Didon, avec le sabre du généralissime Jamont pour manche. (Georges Clemenceau, Vers la réparation, 1899, 391)

Plus intéressant, c'est l'occurrence richissime qui est attesté 102 fois, figurant au $6^{\mathrm{e}}$ rang dans notre liste de fréquences. C'est aussi l'adjectif le plus fréquent parmi ceux qui ont été mentionnés par l'Académie française (voir le chapitre précédent). À la différence des

\footnotetext{
${ }^{8}$ Pour cette variante graphique, voir exemple (13) plus loin.
} 
occurrences qui précèdent, c'est un adjectif assez récent, car les premières occurrences commencent à apparaître dès le XIX ${ }^{\mathrm{e}}$ siècle. Le premier auteur à s'en servir selon Frantext est Honoré de Balzac, décrivant la nouvelle bourgeoisie, ce qui parait essentiel dans l'usage de cet adjectif.

(12) Va, tu seras richissime, ou je perdrai mon nom de César. (Honoré de Balzac, Histoire de la grandeur et de la décadence de César Birotteau, 1837, 53)

On peut ajouter que, souvent, une nuance ironique est présente dans l'usage de cet adjectif et, très souvent, les occurrences réfèrent aux nouveaux-riches. Faisant fonction d'épithète de nature, richissime précède des titres comme marquise, duc, baron, sénateur, banquier, rastaquouère, industriel, collectionneur, femme d'affaires etc.

Quant à la variante graphique rigissime, elle s'explique tout simplement lorsqu'on découvre le contexte dans Splendeurs et misères des courtisanes de Balzac, car c'est la mauvaise prononciation du baron de Nucingen qui est derrière cette variante :

(13) — Ah ! tiaple, s'écria le baron. Irobe, èle ne se vacherait bas t'abbrentre qu'ele tefiient rigissime... Elle héride te sedde milions. (Honoré de Balzac, Splendeurs et misères des courtisanes, 1847, 691)

Sinon, il est évident que les connotations semblent souvent péjoratives en ce qui concerne richissime ; l'exemple tiré de Raymond Roussel (1910) est emblématique à cet égard, référant à un nouveau-riche :

(14) Luxo avait reçu la visite du jeune baron Ballesteros, richissime Argentin qui, depuis plusieurs années, menait en France une vie de folles dépenses et de continuelle ostentation. (Raymond Roussel, Impressions d'Afrique, 1910, 148)

L'adjectif grandissime, qui est attesté dès 1443 chez Charles d'Orléans, n'appelle pas de remarques, mais il est vrai que les occurrences sont plutôt fréquentes, à savoir 87 . Or, ce qu'il convient de remarquer, c'est que les attestations les plus récentes dans Frantext datent de 1986 chez l'auteur belge François de Weyergans (3 occurrences). On se serait peut-être attendu à pouvoir relever des attestations plus tard, mais c'est une recherche vouée à l'échec ; cet adjectif est peut-être en voie de disparition.

(15) Le Grandissime du Budget Indéchiffrable avait tiré les ficelles (François de Weyergans, La Vie d'un bébé, 1986, 44)

Pour les 37 occurrences de amarissime, on notera que toutes les occurrences proviennent de Marguerite de Navarre et de son œuvre Comédie sur le trespas du roy. En fait, elles réfèrent au personnage Amarissime (La Très Amère, à savoir Marguerite) et on ne relève donc pas d'autres occurrences qui pourraient intéresser notre perspective d'inventaire littéraire.

$\mathrm{Au} 11^{\mathrm{e}}$ rang, on relève 16 occurrences de gravissime. Le sens paraît neutre, c'est-àdire sans connotation négative ou positive : "très grave". On notera par exemple quatre occurrences dans Encyclopedie medicale Quillet (1965) qui manquent de mention d'auteur, mais elles relèvent sans doute de la syncrasie de l'auteur de ces passages :

(16) Une urémie aigue gravissime (148) ; affection meningée gravissime (360) ; maladie gravissime (491); urgence gravissime (498) 
Parmi les innovations récentes, on peut citer nullissime. En voici une de Bernfeld (2003), écrivaine qui parle des questions du corps, de troubles de comportement alimentaire, d'identité sexuelle. L'usage de l'adjectif nullissime renforce sans doute le sentiment de désespoir dans ce passage :

(17) L'ambiance de l'appartement, mon allemand nullissime. (Karin Bernfeld, Les portes de l'espérance, 2003, 229)

Autre néologisme, c'est simplissime, dont les quatre occurrences sont datées entre 1982 et 2008. Mais pour ignorantissime, au $13^{\mathrm{e}}$ rang, les exemples contemporains manquent. Cet adjectif figure souvent parmi les exemples cités par les grammairiens tels que Nyrop (1902, 337), et, effectivement, il paraît attesté très tôt ; ensuite, il semble être tombé en désuétude. À l'exception de la fameuse occurrence chez Molière (dans Le Mariage forcé, 1668,31 ), on relève une occurrence chez Pierre de Larivey (1579), dont l'origine italienne joue certainement un rôle à cet égard :

(18) Ah ! ah ! ah ! cet ignorant ignorantissime et sans cervelle me faict rire. (Pierre de Larivey, Le Laquais : comédie, 1579, 120)

Pourtant, il ne s'agit nullement d'ironie chez Jean-François Retz, un des premiers chefs de la fronde, dont l'animosité envers le cardinal Mazarin était bien connu, lorsqu'il emploie cet adjectif :

(19) Le Cardinal Mazarin, ignorantissime en toutes ces matières, dit qu'il s'étonnoit qu'un corps aussi considérable s'amusât à des bagatelles ; (Mémoires : t. 1 : 1613-1648, $1679,297)$

Parmi les cas avec relativement peu d'occurrences, on note, chez Scève, un cas intéressant lorsqu'il se sert d'une réduplication synonymique, puisqu'il se sert de tresbelle et de bellissime, synonymes apparents, dans le même vers. Sans doute la raison de cet usage estelle due à une contrainte métrique, puisque, deux vers plus loin, on relève rarissime.

(20) De corps tresbelle et d'ame bellissime,

Comme plaisir, et gloire à l'Univers,

Et en vertu rarement rarissime

Engendre en moy mille souciz divers :

Mesmes son oeil pudiquement pervers

Me penetrant le vif du sentement

(Maurice Scève, Délie, object de plus haulte vertu, 1544, 288)

Quant à maréchalissime, il est uniquement substantif. L'ironie est apparente dans les cinq occurrences modernes (toutes datant des années 1976 et 1977) : trois sont attestées chez Roy et deux chez Chabrol dont nous reproduisons une de chaque auteur ici :

(21) Les icônes avaient remplacé les portraits de Marx, de Lénine et du Maréchalissime. (Claude Roy, Somme toute, 1976, 107)

(22) la Pravda, suspendu à la place d'honneur, énorme et souriant, il y avait encore le portrait de Staline, l'officiel, tête nue, vareuse de maréchalissime. (Jean-Pierre Chabrol, La Folie des miens, 1977, 361) 
Sinon, un dénominateur commun des occurrences d'adjectifs en -issime est souvent l'influence de l'italien et de l'espagnol même si ces occurrences n'existent pas forcément dans ces langues. Ainsi, le substantif favorissime, dans l'exemple (23) rejoint le substantif campionissimo mentionné auparavant à propos de l'italien et du portugais (dans 2.2) :

(23) angoissée, déroutée soudain par la première chute du Tour de France, qui accablait, une fois de plus, Ocana : un bouledogue néerlandais venait de bouler dans les roues de ce favorissime. (Antoine Blondin, Ma vie entre les lignes, 1982, p. 365)

Dans (24), provenant de T'serstevens (1963), la raison de l'hapax madrilénissime est de toute évidence la liaison avec l'espagnol, où les adjectifs en -ísimo font partie intégrante de la langue depuis longtemps, ce qui n'est pas le cas en français :

(24) au centre nouveau-riche de cette cité redondante : alcala, gran via, bruyante, nourrissante, active, bigarrée, allègre, européenne et madrilénissime avenue, avec ses spectaculaires et surprenantes devantures commerciales (il faut voir ça !), (Albert T'serstevens, L'Itinéraire espagnol, 1963, 219)

Comme les adjectifs en -issime sont souvent des néologismes, on peut parfois assister à une hésitation en ce qui concerne la formation ; d'un côté, simple adjonction de suffixe, d'un autre, forme apocopée (Plénat 2007, 57), deux formes mises à l'index par l'Académie française (voir le chapitre précédent). C'est ainsi qu'on relève sublimissime chez Manoeuvre en 1985 :

(25) Prenez ce pauvre Pete Townshend, des Who. En 1964, il écrit une chanson sublimissime, «My Generation ». (Philippe Manœuvre, L’Enfant du rock, 1985, 217)

et sublissime dix ans plus tard chez Boudard en 1995 :

(26) D'après Alice il devait copuler un peu ailleurs son sublissime baryton. (Alphonse Boudard, Mourir d'enfance, 1995, p. 237)

Nous finirons par citer une innovation basé sur un nom propre, tiré de la Chronique des Pasquier par Georges Duhamel en 1937 (cf. Perko 2007, 466). La valeur négative paraît évidente : le frère aîné Joseph est, comme on le sait, un puissant investisseur ambitieux qui veut se faire élire à l'Académie française.

(27) Malheureusement, Joseph a été intolérable, ou, pour mieux dire, josephissime. (Georges Duhamel, Chronique des Pasquier. 6. Les Maîtres, 1937, 46)

Fait surprenant, c'est l'absence d'adjectifs désignant des couleurs dans cet inventaire ; en principe, nous n'avons relevé qu'une seule occurrence dans Frantext, à savoir rosissime chez Jacques Roubaud (1993) :

(28) Le bébé Juliette avait en 1968-1969, en sa possession, un objet précieux, un rose, un rosissime morceau d'étoffe qu'elle appelait son GnienGnien (majuscule sur chacun des « gnien »). (Jacques Roubaud, La Boucle, 1993, 223)

Ce fait étonne un peu, car on aurait pu imaginer d'autres adjectifs de couleur, mais ce domaine devrait être davantage exploré dans d'autres corpus.

Il ressort de notre inventaire des occurrences du suffixe-issime attestées dans Frantext que même si les écrivains cherchaient autrefois à donner l'image d'une langue parfaite 
comme le signale Siouffi (2010, 405), les occurrences récentes et contemporaines telles que maréchalissime, gravissime, nullissime et simplissime montrent une tentative de quitter ce moule. Selon Perko, le suffixe vit "en marge" du système linguistique, mais il n'est pas vrai pour autant que -issime ne soit pas disponible (2007, 465). Comme la discussion s'est longtemps focalisé autour d'un idiome homogène qui a été compris comme une "langue littéraire", il y a eu entre la grammaire et la littérature un dialogue dont la fonction est de lier étroitement imaginaire linguistique et imaginaire de l'écrit, chose qui sans doute est en train d'évoluer. On constate aussi que seules trois occurrences critiquées par l'Académie française en 2012 se retrouvent dans ce corpus, dominé par des œuvres littéraires.

\section{Occurrences contemporaines}

Nous venons de constater que le nombre d'occurrences dans Frantext est plutôt élevé, ce qui est surprenant, puisqu'on parle de condamnation unanime de ce suffixe par les grammairiens à l'exception des cas où il est employé devant des titres honorifiques. Il ressort de notre étude que la grande majorité d'occurrences appartiennent à la catégorie de titres honorifiques qui sont par conséquent généralement acceptés. Qu'en est-il dans la langue écrite informelle ? On pourrait sans doute s'attendre à des occurrences d'un autre genre que celles que nous venons de voir. La consultation d'un dictionnaire tel que le TLFi nous fournit des renseignements supplémentaires sur ce suffixe : le français a principalement deux bases pour les dérivés avec-issime. Soit il peut s'agir d'un adjectif, ce que nous avons noté auparavant: énormissime, faiblissime, louchissime etc., soit d'un nom propre : béjartissime et godardissime etc. À cet égard, il faut également noter l'emploi croissant de l'allomorphe dit « vulgaire » du suffixe -ique devant -issime, signalé par Plénat (2007,60), qui apparaît surtout sur la Toile. Cet allomorphe semble être utilisé lorsque l'adjectif est disyllabique. Dès lors, on trouve à titre d'exemple classiquissime à côté de classissime, basiquissime, comiquissime, logiquissime, sadiquissime etc. En revanche, quand l'adjectif est trisyllabique, l'allomorphe « savant » est utilisé : on ne trouve donc pas britanniquissime, mais britanissime (Plénat 2007, 60). À l'égard des dérivés formés sur les noms propres, Noailly mentionne non seulement des noms propres de personne tels que saint-laurentissime, chabrolissime et stendhalissime, mais aussi des noms de lieu tels que helvétissime, lyonnissime et nipponissime (1999, 34), ce qui indique une grande diversité quant à l'usage du suffixe. L'usage d'un nom propre affublé du suffixe -issime n'est donc pas sans rappeler celui que nous avons déjà observé à propos de substantifs en italien et en portugais même si nous n'avons pas pu relever des exemples de noms propres dans ces langues encore. Quant à Plénat, il ne manque pas de citer plusieurs dérivés sur des noms propres tels que adjanissime, formé sur Isabelle Adjani, et chiraquissime, formé sur Chirac (2002, 229), exemple également cité par Perko (2007, 466). Dans nos propres relevés sur la Toile, nous avons même pu relever hollandissime, ce qui n'étonne pas :

(29) Hollandissime, cette obsession de «l'équilibre» qui conduit maintenant Manuel Valls à assortir chaque pas à droite d'un autre pas à gauche pour en amortir la charge symbolique et en neutraliser la portée. ${ }^{9}$

\footnotetext{
${ }^{9}$ http://www.lefigaro.fr/vox/politique/2014/04/08/31001-20140408ARTFIG00443-1-occasion-manquee-demanuel-valls.php?pagination $=5$
} 
Le sens de ce suffixe, de connotation péjorative, paraît être "très au centre/milieu". Ainsi, les effets d'humour et d'ironie dépendent aussi du référent du nom de base et de l'énonciation (Perko 2007, 470). Comme les occurrences formées sur la base d'un nom propre semblent assez fréquentes dans la langue informelle, on pourrait s'attendre à relever des occurrences de ce genre sur la Toile ; josephissime dans Frantext (27) en est un bon exemple. D'après Plénat, cet emploi est curieux $(2002,1)$, d'autant plus que la catégorie du dérivé en issime diffère de celle de sa base, mais, on vient de le voir, le phénomène n'est pas sans rappeler les cas en italien dans le domaine des substantifs. Nous avions fait une recherche avancée sur Google sur le suffixe -issime (domaine .fr) en septembre 2016. Cette étude n'est en aucune manière exhaustive, mais donne une bonne idée de néologismes qui apparaissent sur la Toile. Le premier exemple est précisément un cas de nom propre, formé sur l'ancien Président de la République, Jacques Chirac, faisant allusion au soutien qu'a apporté Patrick Sébastien à Jacques Chirac :

(30) Mangez de bonnes soupes, dites-vous "oh non pas lui !" avec Moussier Tombola et Philippe Katerine et retrouvez bien sûr le seul, l'unique, le grand, le chiraquissime Patrick Sébastien $!^{10}$

Notre second exemple est tiré de la presse "people"; les occurrences sont effectivement très fréquentes dans ce genre :

(31) Kylie a exposé sur Instagram son énormissime bague en diamants qui lui a été offerte par Tyga à Noël ! ${ }^{11}$

Autre domaine où le suffixe -issime est attesté, c'est celui de sports. Souvent, on peut relever des titres de presse sous cette rubrique ainsi qu'il ressort de (32), où nous avons affaire à un bel exemple de la surenchère dont fait mention Noailly $(1999,34)$ :

\section{(32) Victoire importantissime pour Union ${ }^{12}$}

Le monde de la musique est le troisième domaine important pour le suffixe qui apparaît dans des titres et des textes des magazines consacrés à la musique contemporaine. L'exemple rock'n'rollissime n'est guère difficile à comprendre, car il désigne le chanteur Leonard Cohen, mais c'est surtout le fait que l'adjectif est un dérivé d'un substantif qui nous intéresse en particulier :

(33) Le poète [Leonard Cohen] a tout essayé : sexe, drogue, retraite monastique. Dépressif, indestructible et fauché, ce survivant qui chantait du folk est rock'n'rollissime. ${ }^{13}$

Mais le suffixe -issime apparaît également dans d'autres domaines plutôt récents. Depuis un certain nombre d'années, ont surgi des phénomènes nouveaux tels que tripadvisor, un guide de voyage qui rassemble les commentaires des clients des restaurants et des hôtels et ceux des voyageurs, remplaçant ou complétant les avis officiels du Guide Michelin par

\footnotetext{
${ }^{10} \mathrm{http} / / /$ ausecoursjeboislatasse.over-blog.com/archive/2014-06/

${ }_{11} \mathrm{http}: / / \mathrm{www}$.public.fr/News/Photos/Photos-Blake-Lively-matez-son-enormissime-bague-de-fiancailles-334185

${ }^{12}$ http://www.lanouvellerepublique.fr/Deux-

Sevres/Sport/Football/Regionaux/n/Contenus/Articles/2013/05/12/Une-victoire-importantissime-1462062

${ }^{13} \mathrm{http} / / / \mathrm{www}$. lepoint.fr/musique/video-les-legendes-du-rock-8-leonard-cohen-l-elegance-du-desespoir-31-122013-1775598 38.php
} 
exemple. Le résultat en est une langue truffée d'adjectifs valorisants, dont le suffixe -issime, de la part des internautes. C'est ainsi qu'on peut relever dans la légende d'une photo :

(34) "Les calamars, topissime !!!!!"14

L'accompagnement de quatre points d'exclamation n'est pas sans intérêt dans ce cas, mais est un signe iconique qui fait partie de la surenchère que nous avons déjà pu constater auparavant. Le même suffixe, topissime, formé sur l'anglicisme top (adjectif), apparaît également dans une autre occurrence dans le magazine des gastronautes www.cookissime.fr (sic) :

(35) Un menu de fêtes topissime ${ }^{15}$

Le cinquième domaine que nous voudrions signaler est celui des sites de caractère sexuel. Il nous semble que les occurrences de ce suffixe y sont très fréquentes, ce qui relève encore une fois de la surenchère. L'exemple suivant, où on reconnaît richissime, qui n'est pas un néologisme d'ailleurs, désigne une vidéo qui promet des fantasmes dans le monde des nantis de la Côte d'Azur :

(36) Richissime femme de Monaco invite un décorateur d'intérieur ${ }^{16}$

Pour finir, les commentaires métatextuels sont une importante source pour les occurrences en -issime, sans doute à cause de l'emphase dans ces commentaires, car il faut dire que, très souvent, ceux-ci sont plutôt négatifs. Il ressort de (37), commentaire fait par un lecteur averti, parait-il, que celui-ci est bien conscient du fait que l'adjectif minabilissime n'existe pas (encore?) dans la langue française :

(37) "une quarantaine de salariés seraiT reclasséE" et non "seraient reclassés" bande d'incompétents! C'est la quarantaine qui s'accorde! Le journalisme rime avec "minabilissime" (mot inexistant) décidément...!!! ${ }^{17}$

Ajoutons que l'usage de trois points d'exclamation paraît traduire l'intonation dans cet exemple ; ainsi, la surenchère, chose que nous avons déjà pu constater auparavant, semble particulièrement fréquente dans les occurrences contemporaines du suffixe -issime.

\section{Remarques finales}

$\mathrm{Au}$ terme de cette étude, où nous nous sommes proposé de décrire l'évolution du produit du suffixe latin -ÍSSIMUS dans les langues romanes et en particulier son rôle dans la langue française, un certain nombre de différences peuvent être constatées en dépit de son histoire commune avec les langues de la péninsule ibérique. Historiquement, la place de cet adjectif dans l'histoire du français diffère de l'italien, notamment parce qu'il s'agit d'un emprunt, sans doute à l'italien, pendant la Renaissance, toutefois rejoignant les langues ibéroromanes. Si cet adjectif apparaît très tôt dans les textes aux $\mathrm{XIV}^{\mathrm{e}}$ et $\mathrm{XV}^{\mathrm{e}}$ siècles, c'est très

\footnotetext{
${ }^{14}$ https://www.tripadvisor.co.za/LocationPhotoDirectLink-g190356-d11607588-i227397478-CDS_MerlLuxembourg_City.html

${ }^{15} \mathrm{http}: / / \mathrm{www}$. cookissime.fr/contents/427

16 http://www.chatounix.com/video/richissime-femme-de-monaco-invite-un-decorateur-d-interieursdyqJji1ryH.html

${ }^{\frac{1}{17}}$ http://plus.lefigaro.fr/article/psa-ferme-lusine-de-dannemarie-20111117-601640/commentaires/7820713
} 
souvent chez des auteurs influencés par l'italien. Autre constatation qui étonne sans doute moins : la plupart des attestations dans Frantext relèvent de la titulature, qui ont été et sont mieux acceptées par les grammairiens que les autres, mais dans ces attestations, une valeur négative ou ironique est souvent présente. On peut aussi constater que, si certaines attestations semblent récentes telles que maréchalissime, gravissime et nullissime, que nous verrons sans doute encore dans l'avenir, d'autres, telles que ignorantissime et grandissime, sont en voie de disparition. D'autres encore, pourtant fréquemment citées dans les grammaires, sont pratiquement inexistantes telles que savantissime. Quant aux occurrences critiquées par l'Académie française, seules quelques-unes se retrouvent dans Frantext.

Une simple recherche sur la Toile montre que dans ces attestations plutôt récentes, il s'agit d'une part de surenchère et d'autre part de néologismes. Très souvent, nous avons affaire à des occurrences formées sur des adjectifs, mais également sur des substantifs. Dans cet emploi, on peut constater que le français rejoint la souplesse de l'italien, où le suffixe peut être ajouté à des substantifs. Ainsi, dans le cas du français, à la différence des langues de la péninsule ibérique et de l'italien, où le produit du suffixe latin -ÍSSIMUS fait partie intégrante de la langue, il faut sans doute parler d'un clivage par rapport à la norme (surtout celle de l'Académie française), entre l'usage officiel et sanctionné, à savoir dans les titres honorifiques, et l'usage populaire, fort présent grâce aux nouveaux médias électroniques. Dans cette langue écrite informelle, les néologismes foisonnent dans plusieurs domaines. Tout comme pendant la période classique, force est de constater que les locuteurs dépassent largement le maigre savoir des grammairiens.

\section{Bibliographie}

Appel, Carl (1930). Provenzalische Chrestomathie mit Abriss der Formenlehre und Glossar. Leipzig: Reisland.

Badía Margarit, Antonio, 1951. Gramática histórica catalana. Barcelona: Noguer.

Bach, Svend \& Schmitt Jensen, Jørgen, 1995 (1990). Større italiensk grammatik. Århus : Munksgaard.

Base textuelle FRANTEXT, ATILF - CNRS \& Université de Lorraine. Site internet : http://www.frantext.fr.

Bosque, Ignacio \& Demonte, Violeta, 1999. Gramática descriptiva de la lengua española, 1, Sintaxis básica de la clases de palabras. Madrid : Espasa.

Bouhours, Dominique, 1675. Remarques nouvelles sur la langue françoise. Paris : Sébastien Mabre-Cramoisy.

Bourciez, Édouard, 1967. Éléments de linguistique romane. Paris : Klincksieck.

Brunot, Ferdinand, 1906. Histoire de la langue française des origines à nos jours, II, Le XVIe siècle. Paris : Colin.

Butt, John \& Benjamin, Carmen, 2011 (1988). A New Reference Grammar of Modern Spanish. Oxon : Hodder.

Dubois, Jean \& Dubois-Charlier, Françoise, 1999. La dérivation suffixale en français. Paris : Nathan.

Elcock, William Dennis, 1960. The Romance Languages. London : Faber \& Faber. 
Estienne, Henri, 1970 (1885). Deux dialogues du nouveau langage françois italianizé. I. Genève: Slatkine Reprints.

Grevisse, Maurice, 2001 (1993). Le bon usage. Grammaire française, refondue par A. Goosse. Paris : Duculot.

Griera, Antonio, 1965. Gramatica historica catalana. Abadia de San Cugat de Valles : Instituto internacional de cultura romanica.

Hultenberg, Hugo, 1903. Le renforcement du sens des adjectifs et des adverbes dans les langues romanes. Thèse pour le doctorat. Upsal : Almqvist \& Wiksell.

Kröll, Heinz, 1963. «Zum "absoluten Superlativ" auf -íssimo im Portugiesischen », in Romanistisches Jahrbuch, XIV, pp. 245-254.

Livet, Charles-Louis, 1859. La grammaire et les grammairiens du XVIe siècle. Paris: Didier.

Mahn, Carl. A. F. (1885). Grammatik und Wörterbuch der altprovenzalischen Sprache. Köthen: Paul Schettler.

Maupas, Charles, 1973 (1618). Grammaire et syntaxe françoise. Genève: Slatkine Reprints.

Meigret, Louis, 1970 (1550). Le tretté de la grammere françoeze. Genève: Slatkine Reprints.

Menéndez Pidal, Ramón, 1968. Manual de gramática histórica española. Madrid: EspasaCalpe.

Noailly, Michèle, 1999. L'adjectif en français. Paris: Ophrys.

Nyrop, Kristian, 1902. Grammaire historique de la langue française. II. Copenhague \& Kristiania : Gyldendalske boghandel, Nordisk forlag.

Perko, Gregor, 2010. « Le suffixe -issime dans le paysage dérivationnel du nom propre en français ». Actes du XXV $V^{e}$ Congrès International de Linguistique et de Philologie Romanes. Danler, P., Siller-Runggaldier, H. \& Iliescu, M. (eds). VII, 465-470.

Plénat, Marc, 2002. «Les contraintes de taille». In B. Fradin, F. Kerleroux et M. Plénat (eds). Aperçus de morphologie du français, pp. 47-63.

Plénat, Marc, 2007. «Jean-Louis Fossat : fossatissime. Note sur la morphophonologie des dérivés en -issime ». In L. Rabassa (ed). Mélanges offerts à Jean-Louis Fossat [Cahiers d'Etudes Romanes (CERCLiD 11-12], pp. 229-248.

Raposo, Eduardo Buzaglo Paiva, Vicente, Graça (eds). 2013. Gramática do português, II, XX: Fundaçâo Calouste Gulbenkian.

Rey, Alain, Duval, Frédéric \& Siouffi, Gilles, 2007. Mille ans de langue française: histoire d'une passion. Paris: Perrin.

Said Ali, Manuel, 1966. Gramática histórica da língua portuguesa. São Paolo: Edições Melhoramentos.

Serianni, Luca, 2008. «Gli italianismi nelle alter lingue romanze : prime riflessioni ». In Italianismi e percorsi dell'italiano nelle lingue latine. Atti del convegno di Treviso, 28 settembre 2007. Paris: Unione latina, pp. 19-41.

Siouffi, Gilles, 2010. Le génie de la langue française. Études sur les structures imaginaires de la description linguistique à l'Âge classique. Paris: Honoré Champion.

Siouffi, Gilles, Luca, 2013. « Bouhours et la notion de 〈 bon usage > ». In Ayres-Bennett, W. \& Seijido, M. (éds). Bon usage et variation sociolinguistique. Perspectives diachroniques et traditions nationales. Paris: ENS Éditions, pp. 77-86.

Solà, Joan, Lloret, Maria-Rosa, Mascaró, Joan \& Pérez Saldanya, Manuel, 2002. Gramàtica del català contemporani, I. Barcelona : Empúries. 
TLFi=Le Trésor de la Langue Française informatisé. Site internet : http://atilf.atilf.fr/tlf.html Wheeler, Max W, Yates, Alan \& Dols, Nicolas, 1999. Catalan : A Comprehensive Grammar. Routledge : London/New York.

Williams, Edwin B., 1938. From Latin to Portuguese. Historical Phonology and Morphology of the Portuguese Language. Philadelphia : University of Pennsylvania Press. 